PROCEEDINGS OF THE

AMERICAN MATHEMATICAL SOCIETY

Volume 131, Number 5, Pages 1347-1355

S 0002-9939(02)06645-5

Article electronically published on September 19, 2002

\title{
CHARACTERIZING NEARLY SIMPLE CHAIN DOMAINS
}

\author{
H. H. BRUNGS AND J. GRÄTER
}

(Communicated by Martin Lorenz)

\begin{abstract}
G. Puninski, using model theoretical methods, showed that if a chain domain $R$ is nearly simple, then $R a+b R=J(R)$ for any nonzero elements $a, b$ in $J(R)$, the Jacobson radical of $R$. Here, an algebraic proof is given for this result, exceptional chain domains are characterized, and it is shown that $V_{0}(R)$, the lattice generated by all proper nonzero left and right ideals, is a direct product of two linearly ordered sets if $R$ is nearly simple. In a certain sense this property characterizes nearly simple chain domains among all integral domains.
\end{abstract}

\section{INTRODUCTION}

Let $K$ be a skew field. A subring $R$ of $K$ is called a chain domain with $K$ as its skew field of quotients (or a chain order in $K$ ) if $x \in K \backslash R$ implies $x^{-1} \in R$. This name is justified by the fact that all left and all right ideals of a chain domain are linearly ordered by inclusion. Such subrings $R$ are sometimes called valuation rings or total subrings of $K$ and are said to have rank one if $J(R)$, the Jacobson radical of $R$, which is always maximal as a left and as a right ideal, is the only nonzero completely prime ideal of $R$.

A chain domain $R$ of rank one is either invariant, i.e., $a R=R a$ for all $a$ in $R$, or it is nearly simple in which case $(0), J(R)$ and $R$ are its only ideals, or $R$ is exceptional and a prime ideal $Q$ exists with $(0) \subset Q \subset J(R)$. In this last case $\bigcap Q^{n}=(0)$ and there are no further ideals between $Q$ and $J(R)$. This was proved for chain domains in [3] and a corresponding result for the wider class of Dubrovin valuation rings was obtained in 2. Examples of nearly simple chain domains were given among others by Mathiak in 9 and in [4 Dubrovin has constructed exceptional chain domains in the rational closure $K$ of the group ring of the universal covering group of $S L(2, \mathbb{R})$.

G. Puninski in [12] used model theoretical methods to investigate modules over a nearly simple chain domain $R$. He proved in Proposition 6.2 that $R a+b R=$ $J(R)$ for all nonzero $a, b$ in $J(R)$. Here we give an algebraic proof of this result in Theorem 2.3, and show in Theorem 2.4 that exceptional chain domains are characterized among the rank one chain domains by the condition that $R a+b R$ is not an ideal of $R$ for some $a, b$ in $R$.

In the final section we show that the results mentioned above are best understood as properties of the lattice $V_{0}(R)$ generated by all proper nonzero left and right

Received by the editors August 6, 2001 and, in revised form, December 17, 2001.

2000 Mathematics Subject Classification. Primary 16L30, 16N60, 16D25; Secondary 06D99.

The first author was supported in part by NSERC. 
ideals of $R$. This lattice is distributive for $R$ a chain domain, and it is a direct product of two nontrivial linearly ordered sets if $R$ is in addition nearly simple. In Theorem 3.7 we characterize all integral domains with this property. Besides nearly simple chain domains there also occur the intersections of two nontrivial subinvariant chain orders which are comaximal.

\section{The Characterization Via $R a+b R$}

Let $R$ be a chain order in $K$ and let $I$ be an ideal (of $R$ ) in $K$, i.e., $I$ is an $R$-subbimodule of $K$. Then

$$
I^{-1}=\{y \in K \mid y I \subseteq R \text { and } I y \subseteq R\} \subseteq K
$$

is also an ideal of $R$.

Lemma 2.1. Let $R$ be a chain order in $K$ and $x$ a nonzero element in $K$. Then $\left(R x^{-1} R\right)^{-1}$ is the largest ideal in $K$ contained in $x R \cap R x$.

Proof. By definition $x^{-1} R\left(R x^{-1} R\right)^{-1} \subseteq R$ and hence $\left(R x^{-1} R\right)^{-1} \subseteq x R$. A similar argument shows $\left(R x^{-1} R\right)^{-1} \subseteq R x$, and $\left(R x^{-1} R\right)^{-1} \subseteq x R \cap R x$ follows. Now let $A$ be an ideal in $K$ with $A \subseteq x R \cap R x$. Then $A \subseteq x R$, hence $x^{-1} A \subseteq R$ and $R x^{-1} R A \subseteq R$ follows. The containment $A R x^{-1} R \subseteq R$ is similarly proved which shows that $A \subseteq\left(R x^{-1} R\right)^{-1}$ and proves the statement of the lemma.

Corollary 2.2. Let $R$ be a chain order in $K$ and let $x, y$ be elements in $K$ with $R x \neq x R$ and $R y \neq y R$. Then the following hold:

(1) $\left(R x^{-1} R\right)^{-1}=\left(R y^{-1} R\right)^{-1} \Longrightarrow R x R=R y R$.

(2) $R x^{-1} R=R y^{-1} R \Longrightarrow R x R=R y R$.

Proof. Let $I_{1}$ be the smallest ideal in $K$ containing $x$, i.e., $I_{1}=R x R$, and let $I_{2}$ be the largest ideal in $K$ which does not contain $x$. Since $R x \neq x R$ it follows that $I_{2}$ is the largest ideal contained in $x R$ and $R x$, i.e., $I_{2}$ is the largest ideal contained in $x R \cap R x$ and $I_{2}$ is distinct from $I_{1}$. Moreover, there are no further ideals between $I_{1}$ and $I_{2}$. Similarly, $I_{2}^{\prime}$, the largest ideal in $K$ contained in $y R \cap R y$, is distinct from the ideal $I_{1}^{\prime}=R y R$ and there are no further ideals between $I_{1}^{\prime}$ and $I_{2}^{\prime}$. To prove 1 ., we observe that $I_{2}=\left(R x^{-1} R\right)^{-1}=\left(R y^{-1} R\right)^{-1}=I_{2}^{\prime}$ by assumption and Lemma 2.1; hence $I_{1}=I_{1}^{\prime}$ as the upper neighbour of $I_{2}$ in the chain of ideals in $K$. The statement 2. follows immediately: $R x^{-1} R=R y^{-1} R$ implies $\left(R x^{-1} R\right)^{-1}=\left(R y^{-1} R\right)^{-1}$ and $R x R=R y R$ by 1 .

To investigate $R a+b R$ for $a, b \in R$ and $R$ a chain order in $K$ we first observe that $R a+b R$ is neither a right nor a left ideal of $R$ in general. Obviously, $R a$ is a right ideal of $a^{-1} R a$ and this means that $R a+b R$ is a right ideal of $a^{-1} R a \cap R$. As an isomorphic image of a chain order, $a^{-1} R a$ is also a chain order in $K$ and we therefore have to deal with the intersection of a finite number of chain orders in $K$. Let us recall some results from [5] (see also [11]): Let $R_{1}, \ldots, R_{n}$ be chain orders in a skew field $K$ and let $S=R_{1} \cap \ldots \cap R_{n}$ be their intersection. Then $S$ is an order in $K$ and any maximal right (left) ideal $M$ of $S$ can be written as $M=J\left(R_{i}\right) \cap S$ for some $i$ where $J\left(R_{i}\right)$ is the unique maximal ideal of $R_{i}$. Furthermore, the classical Ore localization $S_{M}=\left\{s m^{-1} \mid s \in S\right.$ and $\left.m \in S \backslash M\right\}$ is an order in $K$ for any maximal ideal $M$ of $S$ and $S_{M}=R_{i}$ whenever $M=J\left(R_{i}\right) \cap S$. Finally, if $A$ and $B$ are right $S$-submodules of $K$ such that $A R_{i}=B R_{i}$ for $i=1, \ldots, n$, then $A=B$. An immediate consequence of this is that the lattice of all right (left) $S$-submodules 
of $K$ is distributive, since the lattice of all right (left) $R_{i}$-submodules of $K$ is totally ordered by inclusion.

Theorem 2.3 (Puninski). Let $R$ be a nearly simple chain domain and let $a, b$ be nonzero elements in $J(R)$. Then $R a+b R=J(R)$.

Proof. Let $A=R a+b R$. Then $A=a\left(a^{-1} R a\right)+b R$ is a right $S$-submodule of $K$ where $S=a^{-1} R a \cap R$. To prove $A=J(R)$, it is sufficient to show that $A R=J(R)$ and $A\left(a^{-1} R a\right)=J(R) a^{-1} R a$ by the above remark.

First we prove $R a^{-1} R=K$ and we suppose $R a^{-1} R \subset K$. Then there exists $x \in K$ such that $x \notin R a^{-1} R$, i.e., $R a^{-1} R \subset x R$ and $R a^{-1} R \subset R x$. We conclude $x^{-1} R a^{-1} R \subseteq R$ as well as $R a^{-1} R x^{-1} \subseteq R$, and therefore $x^{-1} \in\left(R a^{-1} R\right)^{-1}$. By Lemma 2.1 we know that $\left(R a^{-1} R\right)^{-1}$ is the largest ideal contained in $a R \cap R a$, i.e., $\left(R a^{-1} R\right)^{-1}=(0)$ since $R$ is nearly simple-a contradiction.

From $R a^{-1} R=K$ we get $J(R) a^{-1} R a=J(R) R a^{-1} R a=J(R) K a=K$ and $A\left(a^{-1} R a\right)=A R a^{-1} R a=A K a=K$. Finally, $A R=J(R)$ follows from $R a R \subseteq$ $A R \subseteq J(R)$ and $J(R) \subseteq R a R$ since $R a R$ is a nontrivial ideal and $R$ is nearly simple.

If $R$ is a nearly simple chain domain, then Theorem 2.3 shows that $R a+b R$ is an ideal for all nonzero $a, b$ in $R$. The same is true if $R$ is invariant since then $R a$ and $b R$ are both ideals. The next theorem shows that this statement does not hold for rank one chain orders which are exceptional.

Theorem 2.4. The following conditions are equivalent for a rank one chain order $R$ in $K$ :

(1) $R$ is exceptional.

(2) There exist nonzero elements $a, b$ in $R$ such that $R a+b R$ is not an ideal.

(3) $R$ is not invariant and $R a+b R$ is not an ideal in $R$ whenever both $R a$ and $b R$ are not ideals in $R$ for $a, b$ in $R$.

Proof. We first show that 3. implies 2. Since $R$ is not invariant we obtain $R a \neq a R$ for some $a$ in $R$. If $a R \subset R a$, then $R \subset a^{-1} R a-$ a contradiction since $R$ has rank one. Thus $a R \nsubseteq R a$ and similarly $R a \nsubseteq \nsubseteq a R$. This shows that both $R a$ and $a R$ are not ideals and by 3 . we conclude that $R a+a R$ is not an ideal.

To prove that 2 . implies 1 . we only have to remark that $R a+b R$ is an ideal whenever $R$ is nearly simple or invariant as mentioned above.

It remains to show that 1 . implies 3 . Let $a$ be an element in $R$ such that $R a$ is not an ideal. Then there exist ideals $I_{1}, I_{2}$ in $R$ with $I_{1} \supset R a \supset I_{2}$ and there is no further ideal between $I_{1}$ and $I_{2}$. If $b R$ is contained in $I_{2}$ or contains $I_{1}$, then $R a+b R$ is not an ideal, since both $R a$ and $b R$ are not ideals. We can therefore assume that $I_{1} \supset b R \supset I_{2}$ and $I_{1} \supseteq R a+b R \supset I_{2}$ follows; in addition $R a R=I_{1}=R b R$. Next we prove $I_{2} \neq(0)$. Since $R$ is exceptional by assumption there exists a prime ideal $Q$ such that $(0) \subset Q \subset J(R)$ and $\bigcap Q^{n}=(0)$. Since $a$ and $b$ are nonzero this means $Q^{n} \subseteq R a \cap b R$ for some $n \in \mathbb{N}$ and therefore $(0) \subset Q^{n} \subseteq I_{2}$.

It remains to show that $I_{1} \neq R a+b R=: A$. We consider $A=a a^{-1} R a+b R$ as a right $S$-module where $S=a^{-1} R a \cap R$, and as above $A \neq I_{1}$ if and only if $A R \neq I_{1} R$ or $A a^{-1} R a \neq I_{1} a^{-1} R a$. However, $A R=R a R+b R=I_{1}=I_{1} R$. We will assume that $A a^{-1} R a=I_{1} a^{-1} R a$ and show that this leads to a contradiction. $A a^{-1} R a=R a+b R a^{-1} R a=I_{1} a^{-1} R a$ implies $R+b R a^{-1} R=I_{1} a^{-1} R=I_{1} R a^{-1} R$. 
We apply Corollary 2.2.2 to $x=a^{-1}$ and $y=b^{-1}$ and obtain $R a^{-1} R=R b^{-1} R$. Hence,

$$
b R b^{-1} R=R+b R b^{-1} R=R+b R a^{-1} R=I_{1} R a^{-1} R=I_{1} R b^{-1} R \subseteq R b^{-1} R .
$$

Since $I_{1}$ is an ideal, $b R b^{-1} R=I_{1} R a^{-1} R$ is also an ideal, i.e., $R b R b^{-1} R=I_{1} R b^{-1} R=$ $b R b^{-1} R$. This shows $\left(b R b^{-1} R\right)\left(b R b^{-1} R\right)=b R b^{-1}\left(b R b^{-1} R\right)=b R b^{-1} R$ and hence $b R b^{-1} R$ is an overring of $R$ in $K$. Using $(0) \subset I_{2} \subset R b \cap b R$ we obtain $\left(R b^{-1} R\right)^{-1} \neq$ (0) by Lemma 2.1, i.e., $R b^{-1} R \neq K$ as well as $b R b^{-1} R \neq K$. Since $R$ has rank one it follows that $b R b^{-1} R=R$ which implies $R b^{-1} R b=b^{-1} R b$. But then $R \subseteq b^{-1} R b$ and $R=b^{-1} R b$ since $R$ has rank one. Thus $b R$ is an ideal-a contradiction which proves that 1 . implies 3 .

\section{The lattice $V_{0}(R)$}

In section 2 an algebraic proof of Puninski's theorem was given and we also saw how this characterizes nearly simple chain domains. Now we will apply this result to describe nearly simple chain domains via their lattices generated by all left and right ideals in $K$. Let $R$ be a chain order in $K$. By a right ideal (left ideal) we mean a right (left) $R$-submodule in $K$ and $V(R)$ denotes the lattice generated by all left and right ideals of $R$. Our first result is

Proposition 3.1. $V(R)$ is a distributive lattice for any chain order $R$ in $K$.

Proof. For $A_{1}, A_{2}, A_{3}$ in $V(R)$ it must be shown that

$$
A_{1} \cap\left(A_{2}+A_{3}\right)=\left(A_{1} \cap A_{2}\right)+\left(A_{1} \cap A_{3}\right)
$$

where " $\supseteq$ " is obvious. Thus, let $a$ be in $A_{1} \cap\left(A_{2}+A_{3}\right)$. Only finitely many right or left $R$-ideals in $K$, say $I_{1}, \ldots, I_{m}$, appear in the representations of $A_{1}, A_{2}, A_{3}$. It is easily shown by induction on $m$, that for any $i=1, \ldots, m$ there exists $a_{i} \in I_{i}$ such that $I_{i}^{\prime}:=a_{i} R \subseteq I_{i}$ if $I_{i}$ is a right $R$-ideal or $I_{i}^{\prime}:=R a_{i} \subseteq I_{i}$ otherwise and $a \in A_{1}^{\prime} \cap\left(A_{2}^{\prime}+A_{3}^{\prime}\right)$ where $A_{1}^{\prime}, A_{2}^{\prime}, A_{3}^{\prime}$ are obtained from $A_{1}, A_{2}, A_{3}$ after replacing $I_{1}, \ldots, I_{m}$ by $I_{1}^{\prime}, \ldots, I_{m}^{\prime}$. It follows that $A_{i}^{\prime} \subseteq A_{i}$ for $i=1,2,3$. Let $R_{i}=R$ if $I_{i}^{\prime}$ is a right $R$-ideal and $R_{i}=a_{i}^{-1} R a_{i}$ if $I_{i}^{\prime}$ is not, i.e., $I_{i}^{\prime}=R a_{i}$. In any case $I_{i}^{\prime}$ is a right $R_{i}$-ideal and therefore $A_{1}^{\prime}, A_{2}^{\prime}, A_{3}^{\prime}$ are right ideals of $S$ where $S=R_{1} \cap \ldots \cap R_{m}$. Each $R_{i}$ is a chain order in $K$ and this means that the lattice of all right ideals of $S$ is distributive by the remarks before Theorem 2.3. We get

$$
a \in A_{1}^{\prime} \cap\left(A_{2}^{\prime}+A_{3}^{\prime}\right)=\left(A_{1}^{\prime} \cap A_{2}^{\prime}\right)+\left(A_{1}^{\prime} \cap A_{3}^{\prime}\right) \subseteq\left(A_{1} \cap A_{2}\right)+\left(A_{1} \cap A_{3}\right)
$$

which proves $(*)$.

In the proof of Proposition 3.1 we deal with the intersections of an arbitrary but finite number of conjugates of $R$, and one might wonder whether the intersection $S$ of all $a R a^{-1}$ with $a$ nonzero in $K$ could be considered instead. First of all, $S$ is invariant under all inner automorphisms of $K$, i.e., $a S a^{-1}=S$ for all nonzero $a$ in $K$, and therefore any right $S$-submodule of $K$ is also a left submodule and vice versa. If $V(S)$ denotes the lattice of all $S$-submodules of $K$, then $V(R)$ is a sublattice of $V(S)$. It is still an open problem whether $S$ is an order in $K$ or not. Since $S$ is invariant it has a classical Ore field of fractions but it is not known whether $K$ is the quotient field in general (cf. [1]). In [1] an example is given which shows that $V(S)$ need not be distributive, i.e., the distributivity of $V(R)$ does not carry over to $V(S)$ in general. But there are also nontrivial examples where $V(S)$ is distributive. For instance, in [9] an example of a nearly simple chain domain $R$ is 
given and the intersection $S$ of all its conjugates has a distributive lattice of ideals (cf. [6]).

The next theorem provides a description of all "integral" elements of $V(R)$ for $R$ a nearly simple chain domain. So, let $V_{0}(R)$ denote the sublattice of $V(R)$ which is generated by all nonzero left and right ideals of $R$ contained in $J(R)$.

Theorem 3.2. Let $R$ be a nearly simple chain domain. Then every $A$ in $V_{0}(R)$ can be written uniquely in the form $A=L \cap I$, where $L$ is a nonzero left ideal and $I$ is a nonzero right ideal of $R$ contained in $J(R)$.

Proof. Let $A$ be in $V_{0}(R)$. Then $A$ can be written as the sum of intersections of proper nonzero right or left ideals in $R$ by the distributivity of $V_{0}(R)$. Since the lattice of right ideals of $R$ as well as the lattice of left ideals of $R$ is a chain, it follows that $A=\left(L_{1} \cap I_{1}\right)+\cdots+\left(L_{n} \cap I_{n}\right)$ for nonzero left ideals $L_{i}$ and right ideals $I_{i}$ contained in $J(R)$ for $i=1, \ldots, n$; we can write $L=L \cap J(R)$ for a left ideal $L$ of $R$ contained in $J(R)$ and similarly $I=J(R) \cap I$ for a right ideal $I$. We show next that

$$
\left(L_{1} \cap I_{1}\right)+\left(L_{2} \cap I_{2}\right)=\left(L_{1}+L_{2}\right) \cap\left(I_{1}+I_{2}\right)
$$

for nonzero left ideals $L_{i}$ and nonzero right ideals $I_{i}$ contained in $J(R)$. Using the distributive law we have

$$
\begin{aligned}
\left(L_{1} \cap I_{1}\right)+\left(L_{2} \cap I_{2}\right) & =\left(\left(L_{1} \cap I_{1}\right)+L_{2}\right) \cap\left(\left(L_{1} \cap I_{1}\right)+I_{2}\right) \\
& =\left(L_{1}+L_{2}\right) \cap\left(I_{1}+L_{2}\right) \cap\left(L_{1}+I_{2}\right) \cap\left(I_{1}+I_{2}\right)
\end{aligned}
$$

which is equal to $\left(L_{1}+L_{2}\right) \cap\left(I_{1}+I_{2}\right)$ since $I_{1}+L_{2}=L_{1}+I_{2}=J(R)$ by Theorem 2.3.

It remains to show the uniqueness of the representation $A=L \cap I$. Therefore, assume that $L_{1} \cap I_{1}=L_{2} \cap I_{2}$ for nonzero left ideals $L_{i}$ and right ideals $I_{i}$ in $J(R)$ for $i=1,2$; first also assume $L_{1}=L_{2}=L$. Then

$$
\begin{aligned}
I_{1}=J(R) \cap I_{1} & =\left(L+I_{2}\right) \cap I_{1} \\
& =\left(L \cap I_{1}\right)+\left(I_{2} \cap I_{1}\right) \\
& =\left(L \cap I_{2}\right)+\left(I_{1} \cap I_{2}\right) \\
& =\left(L+I_{1}\right) \cap I_{2}=J(R) \cap I_{2}=I_{2} .
\end{aligned}
$$

Basically, this is the lattice-theoretical proof for the fact that relative complements are unique in a distributive lattice (cf. [7]). To derive the general case, we can assume $I_{2} \subseteq I_{1}$, and since

$$
\begin{aligned}
\left(L_{1}+L_{2}\right) \cap I_{1} & =\left(L_{1}+L_{2}+L_{1}\right) \cap\left(I_{2}+I_{1}\right) \\
& =\left[\left(L_{1}+L_{2}\right) \cap I_{2}\right]+\left[L_{1} \cap I_{1}\right] \quad(\text { see }(+)) \\
& =\left[\left(L_{1}+L_{2}\right) \cap I_{2}\right]+\left[L_{2} \cap I_{2}\right] \\
& =\left(L_{1}+L_{2}+L_{2}\right) \cap\left(I_{2}+I_{2}\right) \\
& =\left(L_{1}+L_{2}\right) \cap I_{2}
\end{aligned}
$$

we get $I_{1}=I_{2}$ and therefore $L_{1}=L_{2}$ by symmetry.

If $\mathfrak{L}_{R}$ denotes the set of all nonzero left ideals of $R$ contained in $J(R)$ and $\mathfrak{R}_{R}$ the corresponding set of right ideals, then $\mathfrak{L}_{R}$ as well as $\mathfrak{R}_{R}$ are totally ordered by inclusion. $\mathfrak{L}_{R} \times \mathfrak{R}_{R}$ is a partially ordered set with respect to

$$
\left(I_{1}, J_{1}\right) \leq\left(I_{2}, J_{2}\right) \Longleftrightarrow\left(I_{1} \subseteq I_{2} \wedge J_{1} \subseteq J_{2}\right)
$$


which turns $\mathfrak{L}_{R} \times \mathfrak{R}_{R}$ into a lattice where

$$
\left(I_{1}, J_{1}\right) \wedge\left(I_{2}, J_{2}\right)=\left(I_{1} \cap I_{2}, J_{1} \cap J_{2}\right),\left(I_{1}, J_{1}\right) \vee\left(I_{2}, J_{2}\right)=\left(I_{1} \cup I_{2}, J_{1} \cup J_{2}\right) .
$$

With this notation Theorem 3.2 states that

$$
\mathfrak{L}_{R} \times \mathfrak{R}_{R} \longrightarrow V_{0}(R),(I, J) \longmapsto I \cap J
$$

is an order isomorphism (cf. (+) in the proof above). Therefore we obtain

Corollary 3.3. If $R$ is a nearly simple chain domain, then $V_{0}(R)$ is a direct product of two nontrivial linearly ordered sets.

Our next goal is to characterize all integral domains with the property mentioned in the corollary above. Here, integral domain means associative ring with $1 \neq 0$ and without zero-divisors. For such a ring $R$ let $V_{0}(R)$ be the lattice generated by all nonzero proper left and right ideals of $R$. Even though $V_{0}(R)$ is generated by elements different from (0) and $R$, the lattice $V_{0}(R)$ may contain $(0)$ or $R$ in general. Following Mathiak (cf. [8]) we introduce

Definition 3.4. A chain order $R$ in a skew field $K$ is called subinvariant if there exists an invariant chain order $S$ in $K$ contained in $R$.

It follows that subinvariant chain orders $R$ in $K$ are exactly the localizations of invariant chain orders of $K$. The next result shows that subinvariant chain orders can also be characterized as those integral domains $R$ with $V_{0}(R)$ a chain.

Theorem 3.5. The following conditions are equivalent for a chain order $R$ in $K$ :

(1) $R$ is subinvariant.

(2) $V_{0}(R)$ is totally ordered by inclusion.

(3) For any nonzero $a$ in $K$ either $R \subseteq a R a^{-1}$ or $a R a^{-1} \subset R$.

Proof. Assume 1. and let $S$ be an invariant chain order contained in $R$. Then $J(R) \subseteq J(S) \subset S \subseteq R$. Any left or right $R$-ideal contained in $J(R)$ is therefore an ideal in $S$ and it follows that $V_{0}(R)$ is totally ordered which proves 2 . If condition 2 holds for $R$, then $R a \subseteq a R$ or $a R \subset R a$ for all nonzero $a$ in $R$, i.e., $R \subseteq a R a^{-1}$ or $a R a^{-1} \subset R$. If $a$ is not in $R$, then $a^{-1}$ is in $R$ since $R$ is a chain order, and we get $R \subseteq a^{-1} R\left(a^{-1}\right)^{-1}$ or $a^{-1} R\left(a^{-1}\right)^{-1} \subset R$, which proves 3 . In order to show that 3 . implies 1. we consider the ring $S=\bigcap a R a^{-1}$, where the intersection is taken over all conjugates of $R$. Then $S$ is an invariant subring of $K$, and we show that $S$ is a chain order in $K$. If $x$ is in $K$ and not in $S$, then $x \notin a R a^{-1}$ for some nonzero $a$ in $K$. But then $x^{-1} \in a R a^{-1}$ and $x^{-1}$ is in any conjugate of $R$ containing $a R a^{-1}$. Any conjugate of $R$ contained in $a R a^{-1}$ also contains $x^{-1}$ since it does not contain $x$. It follows from 3. that the conjugates of $R$ form a chain, hence $x^{-1} \in S$ and $S$ is a chain order in $K$, which proves 1 .

Definition 3.6. Two nontrivial chain orders $R$ and $R^{\prime}$ in $K$ are said to be comaximal if $K$ is the only subring of $K$ which contains $R$ and $R^{\prime}$.

We investigate the intersection $T=R \cap R^{\prime}$ of two nontrivial subinvariant chain orders $R$ and $R^{\prime}$ which are comaximal and claim that $V_{0}(T) \cong \overline{V_{0}(R)} \times \overline{V_{0}\left(R^{\prime}\right)}$ where $\cong$ means isomorphic as lattices, $\overline{V_{0}(R)}=V_{0}(R) \cup\{R\}$, and $\overline{V_{0}\left(R^{\prime}\right)}=V_{0}\left(R^{\prime}\right) \cup\left\{R^{\prime}\right\}$. As in the proof of Proposition 3.1 we conclude that $V_{0}(T)$ is distributive. Thus, any element of $V_{0}(T)$ can be written as a finite sum of elements of the form $I \cap J$ where $I$ is a proper nonzero left and $J$ a right ideal of $T$. Since $I$ is an intersection of a 
left ideal of $R$ and a left ideal of $R^{\prime}$ and similarly for $J$, we see that the elements from $V_{0}(T)$ are finite sums of elements like $I \cap J \cap I^{\prime} \cap J^{\prime}$. Here, $I$ is a nonzero left and $J$ a right ideal of $R$ and $I^{\prime}$ a nonzero left and $J^{\prime}$ a right ideal of $R^{\prime}$, where $I, J$ and $I^{\prime}, J^{\prime}$ may be equal to $R$ or $R^{\prime}$ respectively. $R$ and $R^{\prime}$ are subinvariant and therefore $\overline{V_{0}(R)}$ as well as $\overline{V_{0}\left(R^{\prime}\right)}$ are totally ordered by inclusion. This shows that $V_{0}(T)$ consists of all finite sums of elements of the form $I \cap I^{\prime}$ where $I$ is a nonzero left or right ideal of $R$ and $I^{\prime}$ a nonzero left or right ideal of $R^{\prime}$. With this notation we prove

$$
(++) \quad\left(I \cap I^{\prime}\right)+\left(J \cap J^{\prime}\right)=(I+J) \cap\left(I^{\prime}+J^{\prime}\right) .
$$

By the distributivity of $V_{0}(T)$ we have

$$
\begin{aligned}
\left(I \cap I^{\prime}\right)+\left(J \cap J^{\prime}\right) & =\left[\left(I \cap I^{\prime}\right)+J\right] \cap\left[\left(I \cap I^{\prime}\right)+J^{\prime}\right] \\
& =(I+J) \cap\left(I^{\prime}+J\right) \cap\left(I+J^{\prime}\right) \cap\left(I^{\prime}+J^{\prime}\right) .
\end{aligned}
$$

We are done if $I+J^{\prime}=J+I^{\prime}=K$ is shown and we just prove $I+J^{\prime}=K$. Since $R$ is subinvariant there exists an invariant chain order $S$ in $K$ contained in $R$. Let $S^{\prime}$ be an invariant chain order in $K$ contained in $R^{\prime}$. Then $S$ and $S^{\prime}$ are comaximal since $R$ and $R^{\prime}$ are comaximal and furthermore $I$ is a nonzero ideal of $S$ where $J^{\prime}$ is a nonzero ideal of $S^{\prime}$. Let $a$ and $a^{\prime}$ be nonzero elements in $I$ and $J^{\prime}$ respectively. We show $K \subseteq I+J^{\prime}$. Let $x$ be arbitrary in $K$. By the approximation theorem for comaximal invariant chain domains (cf. [10] or [11]) there exists $y \in K$ such that

$$
x-y \in a S \quad \text { and } \quad y \in a^{\prime} S^{\prime} .
$$

This shows $x=(x-y)+y \in a S+a^{\prime} S^{\prime} \subseteq I+J^{\prime}$ as well as $I+J^{\prime}=K$ and $(++)$ is proved.

From $(++)$ we derive that $V_{0}(T)$ consists of all elements of the form $I \cap I^{\prime}$ where $I$ and $I^{\prime}$ are in $\overline{V_{0}(R)}$ and $\overline{V_{0}\left(R^{\prime}\right)}$ respectively. Now we can proceed as in the proof of Theorem 3.2 to verify that the representation $I \cap I^{\prime}$ is unique. There we used $I+J=J(R)$ for a nonzero left ideal $I$ and a nonzero right ideal $J$ contained in $J(R)$. Here, this argument is replaced by $I+J=K$ for any $I \in \overline{V_{0}(R)}$ and $J \in \overline{V_{0}\left(R^{\prime}\right)}$. Finally, we obtain that

$$
\overline{V_{0}(R)} \times \overline{V_{0}\left(R^{\prime}\right)} \longrightarrow V_{0}(T),\left(I, I^{\prime}\right) \longmapsto I \cap I^{\prime}
$$

is an order isomorphism and this proves one part of

Theorem 3.7. Let $R$ be an integral domain. Then $V_{0}(R)$ is the direct product of two nontrivial linearly ordered sets if and only if $R$ is a nearly simple chain domain or the intersection of two nontrivial subinvariant chain orders in a skew field which are comaximal.

Proof. Let us assume $V_{0}(R) \cong S_{1} \times S_{2}$ where $S_{1}, S_{2}$ are nontrivial linearly ordered sets. Then, $V_{0}(R)$ is distributive and therefore the lattice of all left ideals as well as the lattice of all right ideals are distributive, i.e., $R$ is a $P$-ring in the sense of [5]. Therefore, $R$ has a classical Ore field of fractions $K$, and $R$ is the intersection of chain orders in $K$ which can be obtained from $R$ via localization at the maximal ideals of $R$. We distinguish two cases.

Case 1. The ring $R$ has a unique maximal ideal $M=J(R)$ which implies that $R$ is a chain order in $K$. If $I$ is a nonzero ideal strictly contained in $J(R)$, then $I$ is a waist in $V_{0}(R)$, i.e., $J \subseteq I$ or $I \subseteq J$ for all $J \in V_{0}(R)$. But $V_{0}(R) \cong S_{1} \times S_{2}$, 
where $S_{1}, S_{2}$ are nontrivial, shows that this cannot occur. Therefore, $R$ has rank one and $R$ cannot be invariant or exceptional.

Case 2. The ring $R$ has more than one maximal ideal. Then $R$ belongs to $V_{0}(R)$ and $S_{1} \times S_{2}$ has a maximal element which means that $S_{i}$ has a maximal element $s_{i}, i=1,2$. Let $M_{1}$ be a maximal ideal of $R$. Then $M_{1}$ is maximal as a right and left ideal. Therefore, $M_{1}$ is a lower neighbour of $R$ in $V_{0}(R)$ and if $M_{1}$ corresponds to $\left(x_{1}, x_{2}\right)$ in $S_{1} \times S_{2}$ we can assume $x_{2}=s_{2}$ and $x_{1}=t_{1}$ is the lower neighbour of $s_{1}$ in $S_{1}$. We conclude that $R$ has only two maximal ideals, $M_{1}$ and $M_{2}$, and $M_{2}$ corresponds to $\left(s_{1}, t_{2}\right)$ where $t_{2}$ is the lower neighbour of $s_{2}$. Since $V_{0}(R)$ is distributive, the localization $B_{i}=R_{M_{i}}={ }_{M_{i}} R$ is a chain order in $K, i=1,2$. Furthermore, $R=B_{1} \cap B_{2}$ and $B_{1}, B_{2}$ are comaximal. Otherwise there exists a nonzero completely prime ideal $P$ of $R$ which is a waist in $V_{0}(R)-$ a contradiction. We show that $B_{i}$ is subinvariant and we can assume $i=1$. Let $a$ be a nonzero element in $J\left(B_{1}\right)$ and let $P$ be the minimal completely prime ideal of $B_{1}$ containing $a$. The nonzero completely prime ideal $Q=P \cap R$ satisfies $Q \subseteq J\left(B_{1}\right) \cap R$ and $Q \nsubseteq J\left(B_{2}\right) \cap R$ by the comaximality of $B_{1}, B_{2}$. There exists $x \in Q$ such that $x \notin J\left(B_{2}\right) \cap R$ and $x$ is a unit in $B_{2}$. In the representation $S_{1} \times S_{2}$ the left ideal $R x$ and the right ideal $x R$ correspond to elements $\left(, s_{2}\right)$ which means $x R \subseteq R x$ or $R x \subseteq x R$. Let $x R \subseteq R x$ and the case $R x \subseteq x R$ can be treated similarly. Then $R \subseteq x^{-1} R x$ and $B_{2}=x^{-1} B_{2} x$ as $x$ is a unit in $B_{2}$. Since $x^{-1} R x=x^{-1} B_{1} x \cap x^{-1} B_{2} x$ and $x^{-1} B_{1} x, x^{-1} B_{2} x$ are comaximal, we conclude $B_{1} \subseteq x^{-1} B_{1} x$ and $B_{1} x$ is an ideal of $B_{1}$ contained in $P$ which is the minimal completely prime ideal of $B_{1}$ containing $a$. The intersection $\bigcap_{n}\left(B_{1} x\right)^{n}$ is a completely prime ideal of $B_{1}$ strictly contained in $P$ and there exists $n \in \mathbb{N}$ such that $B_{1} x^{n}=\left(B_{1} x\right)^{n} \subset B_{1} a$ and $R \cap B_{1} x^{n} \subseteq R \cap B_{1} a$. Clearly, $x \notin R \cap J\left(B_{2}\right)$ implies $R \cap B_{1} a \nsubseteq R \cap J\left(B_{2}\right)$. There exists $u \in R \backslash J\left(B_{1}\right)$ and $v \in R$ such that $a=u^{-1} v$ and this means $B_{1} a=B_{1} u^{-1} v=B_{1} v$. If $v \notin J\left(B_{2}\right)$, the arguments we used above to investigate $x$ now show that $v B_{1} \subseteq B_{1} v$ or $B_{1} v \subseteq v B_{1}$. Since $u$ is a unit in $B_{1}$, this finally implies $a B_{1} \subseteq B_{1} a$ or $B_{1} a \subseteq a B_{1}$. If $v \in J\left(B_{2}\right)$, then $B_{1}\left(x^{n}+v\right)=B_{1} v$ since $B_{1} x^{n} \subset B_{1} v$, and $B_{2}\left(x^{n}+v\right)=B_{2}$ since $B_{2} v \subset B_{2} x^{n}=B_{2}$ and we consider $x^{n}+v$ instead of the element $v$.

Theorem 3.7 shows how nearly simple chain domains can be characterized by the lattice generated by all proper nonzero left and right ideals.

Corollary 3.8. Let $R$ be an integral domain and let $V_{0}(R)$ be the lattice which is generated by all proper nonzero left and right ideals in $R$. Then $R$ is a nearly simple chain domain if and only if $V_{0}(R)$ is the direct product of two nontrivial linearly ordered sets and $V_{0}(R)$ has no dual atoms.

Proof. If $V_{0}(R)$ is the direct product of two nontrivial linearly ordered sets, then the supremum $s$ of $V_{0}(R)$ exists in $V_{0}(R)$, as we observed above. If $R$ is not nearly simple, then $R$ is the supremum and any maximal ideal $M$ is a lower neighbour of $s$ which is called a dual atom. If $R$ is nearly simple, then $J(R)$ is the supremum of $V_{0}(R)$ and there is no left or right ideal which is a lower neighbour of $J(R)$.

\section{REFERENCES}

1. H.H. Brungs and J. Gräter, Value Groups and Distributivity, Can. J. Math. 43 (1991), 11501160. MR 93a:16016

2. H.H. Brungs, H. Marubayashi and E. Osmanagic, A classification of prime segments in simple artinian rings, Proc. Amer. Math. Soc. 128 (2000), 3167-3175. MR 2001b:16055 
3. H.H. Brungs and G. Törner, Chain rings and prime ideals, Arch. Math. 27 (1976), 253-260. MR 54:7537.

4. N.I. Dubrovin, The rational closure of group rings of left orderable groups, Mat. Sbornik 184(7) (1993), 3-48. MR 94g:16035

5. J. Gräter, Zur Theorie nicht kommutativer Prüferringe, Archiv der Mathematik 41 (1983), 30-36. MR 85e:16007

6. J. Gräter, On noncommutative Prüfer rings, Archiv der Mathematik 46 (1986), 402-407. MR 87h:16005

7. G. Grätzer, General Lattice Theory, Academic Press (1978). MR 80c:06001b

8. K. Mathiak, Bewertungen nicht kommutativer Körper, J. Algebra 48 (1977), 217-235. MR 58:5614

9. K. Mathiak, Zur Bewertungstheorie nicht kommutativer Körper, J. Algebra 73 (1981), 586600. MR 83c: 12026

10. K. Mathiak, Der Approximationssatz für Bewertungen nicht kommutativer Körper, J. Algebra 76 (1982), 280-295. MR 83m:12044

11. K. Mathiak, Valuations of Skew Fields and Projective Hjelmslev Spaces, LNM 1175, Springer (1986). MR 87g:16002

12. G. Puninski, Some model theory over a nearly simple uniserial domain and a decomposition of serial modules, J. Pure Appl. Algebra 163 (2001), 319-337.

Department of Mathematical Sciences, University of Alberta, Edmonton, Alberta, Canada T6G 2G1

E-mail address: hbrungs@math.ualberta.ca

Institut für Mathematik, Universität Potsdam, Postfach 601553, 14469 Potsdam, GERMANY

E-mail address: graeter@rz.uni-potsdam.de 